\title{
Association between Nod-like receptor protein 3 inflammasome and gouty nephropathy
}

\author{
YAN-ZI ZHANG, XIAO-LU SUI, YUN-PENG XU, FENG-JUAN GU, AI-SHA ZHANG and JI-HONG CHEN \\ Department of Nephrology, Affiliated Baoan Hospital of Shenzhen, The Second School of Clinical Medicine, \\ Southern Medical University, Shenzhen, Guangdong 518000, P.R. China
}

Received October 15, 2018; Accepted July 12, 2019

DOI: $10.3892 /$ etm.2020.8694

\begin{abstract}
Crystalized deposits of monosodium urate activate the Nod-like receptor protein $3\left(\mathrm{NLRP}_{3}\right)$ inflammasome, resulting in kidney damage. The present study investigated whether the $\mathrm{NLRP}_{3}$ inflammasome is associated with the progression of hyperuricaemia and gouty nephropathy. Adult male patients were recruited at the Affiliated Baoan Hospital of Shenzhen and divided into three groups of 15 patients each: The control group, the hyperuricaemia group and the gouty nephropathy group. General characteristics and organ function indicators were also measured for each patient. $\mathrm{NLRP}_{3}$, apoptosis-associated speck like protein (ASC) and caspase-1 mRNA and protein expressions in peripheral blood mononuclear cells were detected. The expression of certain downstream inflammatory factors, including interleukin (IL)-1 $\beta$ and IL-18 were also assessed in plasma. The results demonstrated that the concentration of uric acid and creatinine were increased in the hyperuricaemia and gouty nephropathy groups compared with the control group. $\mathrm{NLRP}_{3}$, ASC and caspase- 1 mRNA and protein expression, and IL- $1 \beta$ and IL-18 expression were increased in the hyperuricaemia and gouty nephropathy groups compared with the control group. In addition, ASC and caspase-1 mRNA and protein expression, and IL-1 $\beta$ expression were higher in the gouty nephropathy group compared with the hyperuricaemia group. In conclusion, the present results supported the hypothesis that the $\mathrm{NLRP}_{3}$ inflammasome signalling pathway is associated with gouty nephropathy leading to initiation of the inflammatory response and causing renal damage.
\end{abstract}

\section{Introduction}

The role of uric acid in the causation and progression of chronic kidney disease (CKD) has been debated; however,

Correspondence to: Dr Ji-Hong Chen, Department of Nephrology, Affiliated Baoan Hospital of Shenzhen, The Second School of Clinical Medicine, Southern Medical University, 118 Longjing Road, Xinan Street, Baoan, Shenzhen, Guangdong 518000, P.R. China

E-mail: chenjihong0606@hotmail.com

Key words: gouty nephropathy, hyperuricaemia, Nod-like receptor protein 3 inflammasome uric acid has been reconsidered as a potential contributory risk factor for the development and progression of CKD over the last 15 years (1-2). Excessive uric acid, usually in the form of monosodium urate (MSU) crystals, precipitates in synovial cavities and other anatomic location to induce severe inflammation and debilitating pain (3-6). In particular, deposits of uric acid in the kidney causes gouty nephropathy, which is the most serious complication of hyperuricemia (3-6). It is considered that the activation of renin-angiotensin, inflammatory factors, endothelial dysfunction and cyclooxygenase-2 (COX-2) serve important roles in gouty nephropathy $(5,6)$. The identified underlying mechanisms of gouty nephropathy have revealed that inflammation has a dominant role in its pathogenesis.

The Nod-like receptor protein $3\left(\mathrm{NLRP}_{3}\right)$ inflammasome is comprised of $\mathrm{NLRP}_{3}$, apoptosis-associated speck like protein (ASC) and caspase-1 and is the most extensively studied inflammasome of recent years. It is involved in certain human inflammatory and autoimmune diseases, including cryopyrin-associated periodic syndrome, ischaemia reperfusion injury and atherosclerosis $(7,8)$. Following detection of cellular stress, $\mathrm{NLRP}_{3}$ oligomerizes by homotypic interactions between NACHT domains. The pyrin domains (PYD) of $\mathrm{NLRP}_{3}$ then becomes exposed for ASC binding. The caspase activation and recruitment domains (CARD) of ASC in turn recruits pro-caspase-1 through CARD-CARD interactions (9). Following $\mathrm{NLRP}_{3}$ inflammasome formation, procaspase- 1 is converted to active caspase- 1 , which regulates the maturation of proinflammatory cytokines, including interleukin (IL)- $1 \beta$ and IL-18 (10), further aggravating renal damage.

MSU crystals cause the formation of inflammasomes in vivo (11). In addition, the inflammatory effect of MSU crystals is primarily mediated by $\mathrm{NLRP}_{3}$ inflammasomes driving the production of IL- $1 \beta$ and IL-18. IL-1 $\beta$ is likely the main agent that triggers systemic inflammation (3). Therefore, these observations prompted the present study to assess the role of the $\mathrm{NLRP}_{3}$ inflammasome in the mediation of the innate immune inflammatory response to MSU crystal deposition with regards to gouty nephropathy. The present study investigated the role of the $\mathrm{NLRP}_{3}$ inflammasome signalling pathway with the progression of hyperuricemia and gouty nephropathy, the results of which may provide a novel theoretical basis and therapeutic target for the early prevention and treatment of gouty nephropathy. 


\section{Materials and methods}

Study subjects. A total of 45 male patients (18-70 years old) were recruited at the People's Hospital of Shenzhen Baoan between July 2016 and December 2017. According to the inclusion and exclusion criteria, these patients were divided into three groups $(n=15)$ : The control group, the hyperuricaemia group and the gouty nephropathy group. The present study was approved by the Ethics Committee of the Affiliated Bao'an Hospital of Shenzhen (approval no. BYL2016001). Written informed consent was obtained from all participants.

Inclusion criteria. Patients in the control group received a health examination. There were no abnormalities in the laboratory indicators of the selected subjects and patients had no history of cardiovascular disease or liver disease (including diabetes and gout). Patients also had no presence of infection or autoimmune disease. Hyperuricaemia was defined as levels of serum uric acid $>6-7 \mathrm{mg} / \mathrm{dl}$ (12). The diagnosis of gouty nephropathy was based on the diagnosis of primary gout (13), with one or more of the following parameters: Urinary protein $>150 \mathrm{mg} / \mathrm{dl}$; urine white blood cells $>5 /$ high power field (HPF); urine red blood cells $>3 /$ high power field; serum creatinine $>115 \mu \mathrm{mol} / 1$; blood uric acid/creatinine ratio $>2.5$; ultrasound or ureterography revealing renal calculus and kidney shrinkage. All of the aforementioned cases excluded urinary tract infections and other diseases such as cancer.

Exclusion criteria. Exclusion criteria was based on previous literature (14) and was as follows: female; $<18$ years old or $>70$ years old; patients with secondary hyperuricaemia or stage 4-5 chronic kidney disease; acute hyperuricaemia and the presence of acute renal function deterioration factors; patients with severe cardiovascular disease, liver and kidney disease, lung disease, fractures, tumors, infectious and autoimmune disease, and mental illness; diseases that may affect $\mathrm{NLRP}_{3}$ inflammasome signalling pathways; patients who had been using uric acid drugs outside the hospital or had been treated with lipid-lowering drugs or anti-inflammatory and anti-oxidative drugs during the 4 weeks prior to admission.

Detection of organ function indicators. Biochemical serum and urine samples were obtained following $8 \mathrm{~h}$ fasting. A total of $15 \mathrm{ml}$ serum sample was collected from each patient and shipped to the Laboratory Services at the Affiliated Bao'an Hospital of Shenzhen (Guangdong, China) for biochemical analysis, which was obtained by centrifugation at $500 \mathrm{xg}$ for $10 \mathrm{~min}$ at $4^{\circ} \mathrm{C}$. Urinary biochemical parameters were measured from the patients' first morning urine sample. Other standard parameters were measured including blood and urine analysis. The parameters tested for blood included white blood cell (WBC), red blood cell (RBC), hemoglobin (HB), platelets (PLT), total cholesterol (TC), total triglycerides (TG), low-density lipoprotein (LDL), high-density lipoprotein (HDL), total protein (TP), albumin (ALB), alanine aminotransferase (ALT), aspartate aminotransferase (AST), serum creatinine (Cr), uric acid (UC), blood urea nitrogen (BUN), prothrombin time (PT), activated partial thromboplastin time (APTT), thrombin time (TT) and fibrinogen (FIB). Other parameters tested for urine were urine leukocytes, urine erythrocytes and urine protein.
Isolation and culture of peripheral blood mononuclear cells. Centrifugation (speed, 1,600 x g; duration, $10 \mathrm{~min}$; temperature, $4^{\circ} \mathrm{C}$ ) was performed on blood samples in an EDTA tube to obtain the buffy coat. Samples were stored at $-80^{\circ} \mathrm{C}$ for future use. Peripheral blood mononuclear cells were isolated with Ficoll-Paque Plus (GE Healthcare). Monocytes were isolated by magnetic bead negative selection according to the instructions of Dynabeads ${ }^{\circledR}$ Untouched $^{\mathrm{TM}}$ Human Monocytes kit (Invitrogen; Thermo Fisher Scientific, Inc.).

Isolated monocyte lysates were separated from which $5 \mu 1$ was extracted for viability verification. Trypan Blue staining confirmed that the viability of the cells was $>95 \%$. Cell concentration was then adjusted to $5 \times 10^{5}$ cells $/ \mathrm{ml}$ using a hemocytometer using the following formula: Number of cells $/ \mathrm{ml}=$ number of cells counted in 100 small grids $/ 100 \times 400 \times 10,000 x$ dilution factor. The total number of monocytes was counted to be $34.311 \pm 19.912 \times 10^{5}$. A third of which was used for RNA extraction, whilst the rest were used for western blotting. The number of monocytes for RNA extraction and western blot analysis were divided 1:2. A total of $200 \mu \mathrm{l}$ TRIzol (Invitrogen; Thermo Fisher Scientific, Inc.) was added to monocytes for RNA extraction, whereas 30-50 $\mu 1$ PBS (Invitrogen; Thermo Fisher Scientific, Inc.) was added for western blotting experiments.

Reverse transcription-quantitative PCR (RT-qPCR) to determine $\mathrm{NLRP}_{3}, \mathrm{ASC}$ and caspase-1 mRNA expression in peripheral blood mononuclear cells. Total RNA was extracted from monocytes using TRIzol solution (Invitrogen; Thermo Fisher Scientific, Inc.) according to the manufacturer's protocol. RNA concentration and purity were assessed via electrophoresis and a UV spectrophotometer (Thermo Fisher Scientific, Inc.), respectively. RNA was reverse transcribed to cDNA following the protocol provided by the RevertAid First Strand cDNA Synthesis kit (Thermo Fisher Scientific, Inc.). cDNA was stored at $-20^{\circ} \mathrm{C}$ prior to qPCR amplification using the TransStart ${ }^{\circledR}$ Tip Green qPCR Supermix $2 X$ kit (TransGen Biotech Co., Ltd.), using the SYBR-Green I fluorophore, according to manufacturer's protocol. All qPCR reactions were performed in an Applied Biosystems 7500 system (Applied Biosystems; Thermo Fisher Scientific, Inc.) using the following thermocycling conditions: Initial denaturation at $95^{\circ} \mathrm{C}$ for $30 \mathrm{sec}$, followed by 40 cycles of $95^{\circ} \mathrm{C}$ for $5 \mathrm{sec}$ and $60^{\circ} \mathrm{C}$ for $30 \mathrm{sec}$. Finally, the melting curve was generated (dissociation, $60^{\circ} \mathrm{C}$ at $30 \mathrm{sec}$ and $95^{\circ} \mathrm{C}$ at $15 \mathrm{sec}$ ) to determine normality. Gene expression was calculated using the $2^{-\Delta \Delta \mathrm{Cq}}$ method (15). $\beta$-actin was used as an internal reference for the mRNA levels of $\mathrm{NLRP}_{3}, \mathrm{ASC}$ and caspase-1 detected. The primer sequences used for qPCR are presented in Table I.

Western blot analysis to determine $N L R P_{3}, A S C$ and caspase-1 protein expression in peripheral blood mononuclear cells. Cells were lysed using ice-cold RIPA lysis buffer (Beijing Solarbio Science \& Technology Co., Ltd.) and protein concentration was quantified using Bicinchoninic Acid Assay kit (Thermo Fisher Scientific, Inc.). Protein samples $(10 \mu \mathrm{g})$ were separated by 8,10 or $12 \%$ SDS-PAGE before being transferred to PVDF membranes. The membranes were then blocked with 5\% milk dissolved in TBS supplemented with $1 \%$ Tween-20 at room temperature for $1 \mathrm{~h}$, prior to incubation with 
Table I. Primer sequences.

\begin{tabular}{lll}
\hline Gene & \multicolumn{1}{c}{ Forward primer } & \multicolumn{1}{c}{ Reverse primer } \\
\hline$\beta$-actin & 5'-AACCGCGAGAAGATGACCCAGAT-3' & 5'-GGATAGCACAGCCTGGATAGCA-3' \\
$\mathrm{NLRP}_{3}$ & 5'-ATGGGTTTACTGGAGTACCTTTC-3' & 5'-CTGTCTTCAATGCACTGGAATCTG-3' \\
ASC & 5'-GATGCTCTGTACGGGAAGGTC-3' & 5'-TCCAGTTCCAGGCTGGTGT-3' \\
Caspase-1 & 5'-GGAAGACTCATTGAACATATGCAAG-3' & 5'-CTTGTCAAAGTCACTCTTTCAGTG-3'
\end{tabular}

$\mathrm{NLRP}_{3}$, nucleotide-binding domain and leucine-rich repeat protein 3; ASC, apoptosis-associated speck like protein.

Table II. General characteristics of enrolled patients.

\begin{tabular}{lccc}
\hline Characteristics & $\begin{array}{c}\text { Control } \\
(\mathrm{n}=15)\end{array}$ & $\begin{array}{c}\text { Hyperuricemia } \\
(\mathrm{n}=15)\end{array}$ & $\begin{array}{c}\text { Gouty nephropathy } \\
(\mathrm{n}=15)\end{array}$ \\
\hline Age (years) & $45.20 \pm 10.19$ & $48.27 \pm 14.91$ & $48.3 \pm 11.5$ \\
Height $(\mathrm{m})$ & $1.72 \pm 0.06$ & $1.70 \pm 0.05$ & $1.66 \pm 0.05$ \\
Body weight $(\mathrm{kg})$ & $72.25 \pm 6.97$ & $69.98 \pm 12.08$ & $61.39 \pm 6.83$ \\
BMI $\left(\mathrm{kg} / \mathrm{m}^{2}\right)$ & $24.28 \pm 1.53$ & $24.38 \pm 4.53$ & $22.19 \pm 2.43$ \\
SBP $(\mathrm{mmHg})$ & $116.00 \pm 7.61$ & $115.93 \pm 9.83$ & $121.33 \pm 10.87$ \\
DBP $(\mathrm{mmHg})$ & $79.27 \pm 7.98$ & $76.47 \pm 7.60$ & $74.80 \pm 8.78$ \\
FBG $(\mathrm{mmol} / \mathrm{l})$ & $4.73 \pm 0.45$ & $5.17 \pm 0.70$ & $4.77 \pm 0.39$ \\
\hline
\end{tabular}

Values are presented as the mean \pm standard deviation. BMI, body mass index; SBP, systolic blood pressure; DBP, diastolic pressure; FBG, fasting blood-glucose.

primary antibodies against NLRP (1:300; cat. no. sc-134306; Santa Cruz Biotechnology, Inc.), caspase-1 (1:300; cat. no. sc-622; Santa Cruz Biotechnology, Inc.), ASC (1:300; cat. no. sc-514414; Santa Cruz Biotechnology, Inc.) or GAPDH (1:1,000; cat. no. A01020; Abbkine, Inc.) diluted in PBS supplemented with $0.1 \%$ Tween-20 (PBST) and $1 \%$ bovine serum albumin [Sangon Biotech (Shanghai) Co., Ltd.] at $4^{\circ} \mathrm{C}$ overnight. Subsequently, the PVDF membranes were incubated with horseradish peroxidase-conjugated goat anti-mouse (1:1,000; cat.no. A0216; Beyotime Institute of Biotechnology) or goat anti-rabbit (1:10,000; cat. no. A21020-1; Abbkine, Inc.) secondary antibodies diluted in PBST supplemented with $5 \%$ milk at room temperature for $1 \mathrm{~h}$ with agitation. The proteins were then visualized using Pierce ${ }^{\mathrm{TM}}$ ECL Western Blotting Substrate (Thermo Fisher Scientific, Inc.) and all bands were analysed using Gel Doc ${ }^{\mathrm{TM}} \mathrm{XR}+$ Gel Documentation system (Bio-Rad Laboratories, Inc.) to calculate the densitometric values.

ELISA for the determination of IL-1 $\beta$ and IL-18 levels in plasma. IL-1 $\beta$ (cat. no. 70-EK101B2) and IL-18 (cat. no. 70-EK1182) ELISA kits [Hangzhou Multi Sciences (Lianke) Biotech Co., Ltd.] were used to determine the concentration of IL-1 $\beta$ and IL-18 in $2 \mathrm{ml}$ total plasma, according to manufacturer's protocol.

Statistical analysis. Each experiment was repeated three times. All data were analysed using SPSS v.17.0 (SPSS, Inc.) software and expressed as the mean \pm standard deviation.
One-way analysis of variance was performed to compare the mean of multiple groups and a least significant difference post hoc test was used. $\mathrm{P}<0.05$ was considered to indicate a statistically significant difference.

\section{Results}

Kidney function is damaged in hyperuricaemia and gouty nephropathy groups. Patient general characteristics are provided in Table II, Figs. 1 and 2. The expression of uric acid (Fig. 1C), blood urea nitrogen (Fig. 1B) and blood lipids (Fig. 1F) including total cholesterol, total triglycerides and low-density lipoprotein was higher in the hyperuricaemia and gouty nephropathy groups compared with the control group, but the level of high-density lipoprotein exhibited the opposite trend. Levels of urine leukocytes and erythrocytes were higher in the gouty nephropathy group compared with both the control and hyperuricemia groups (Fig. 2E-F). Serum creatinine (Fig. 1A), uric acid (Fig. 1C), blood urea nitrogen (Fig. 1B) were greater in the gouty nephropathy group and hyperuricaemia group compared with the control group. Serum creatinine and urine protein are common indicators of renal function and used to monitor the progression of chronic kidney disease (16). These results demonstrated that the kidney had been further damaged in the progression from hyperuricaemia to gouty nephropathy.

Successful peripheral blood mononuclear cell isolation. Peripheral blood mononuclear cells were isolated from the 
A

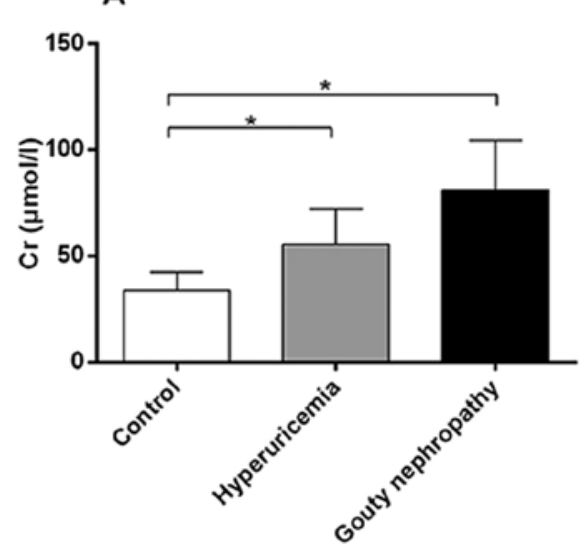

C
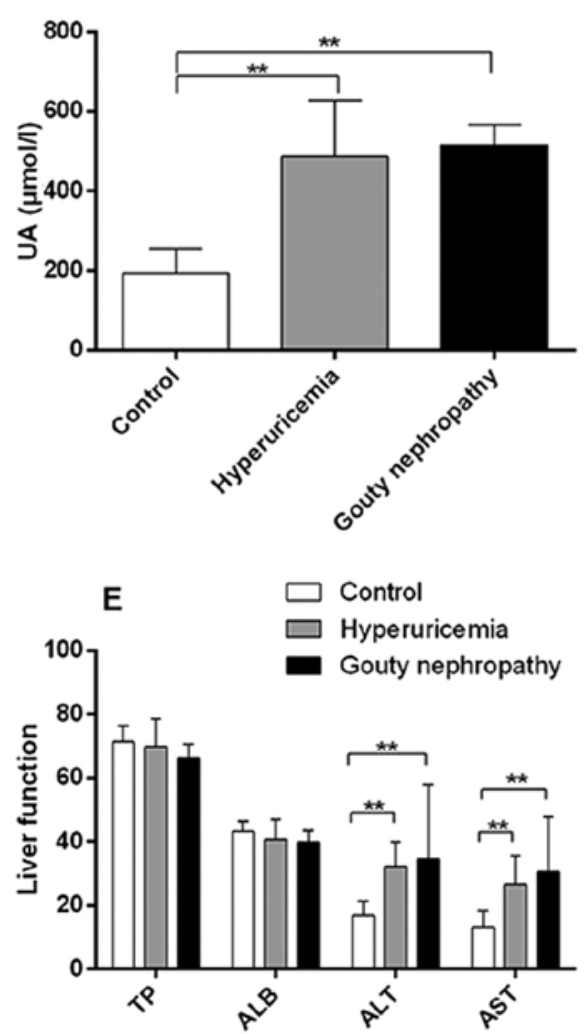

B

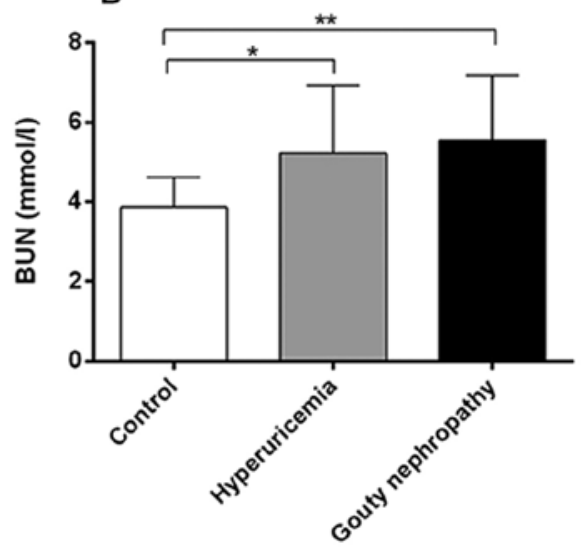

D
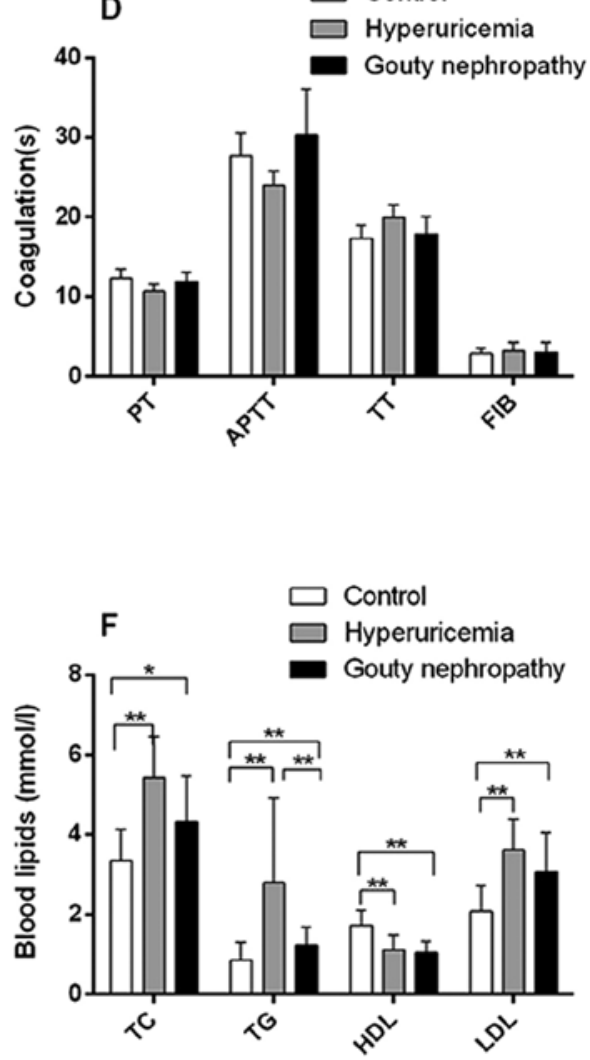

Figure 1. Organ function indicators of enrolled patients, including renal and liver function, coagulation and blood lipids. (A) Levels of Cr, (B) BUN, (C) UA, (D) coagulation functions, (E) liver function and (F) blood lipids of patients in the control, hyperuricemia and gouty nephropathy groups. ${ }^{*} \mathrm{P}<0.05$ and ${ }^{* *} \mathrm{P}<0.01$, with comparisons indicated by lines. Cr, creatinine; BUN, blood urea nitrogen; UA, uric acid; PT, prothrombin time; APTT, activated partial thromboplastin time; TT, thrombin time; FIB, fibrinogen; TP, total protein; ALB, albumin; ALT, alanine aminotransferase; AST, aspartate aminotransferase; TC, total cholesterol; TG, total triglycerides; HDL, high density lipoprotein; LDL, low density lipoprotein.

peripheral blood of 45 patients for in vitro experiments. Trypan blue staining (Fig. 3) confirmed that $>95 \%$ were viable cells (Table III).

$\mathrm{NLRP}_{3}, \mathrm{ASC}$ and caspase-1 mRNA expression are increased in the hyperuricaemia and gouty nephropathy groups. $\mathrm{NLRP}_{3}, \mathrm{ASC}$ and caspase-1 mRNA levels were significantly increased in the hyperuricaemia and gouty nephropathy groups compared with the control group (Table IV). Compared with the hyperuricaemia group, the gouty nephropathy group had a significantly higher expression of ASC and caspase-1 mRNA (Table IV). The mRNA expression in peripheral blood mononuclear cells demonstrated that the $\mathrm{NLRP}_{3}$ inflammasome may serve a pivotal role in gouty nephropathy.

Expression of $\mathrm{NLRP}_{3}, \mathrm{ASC}$, caspase-1 protein increases in the hyperuricaemia and gouty nephropathy group. Gouty nephropathy group had a significantly higher expression of $\mathrm{NLRP}_{3}, \mathrm{ASC}$ and caspase-1 protein compared with the control group (Table V). The expression of ASC and caspase-1 protein was higher in the gouty nephropathy group compared with the hyperuricaemia group (Table V; Fig. 4). Peripheral blood mononuclear cell protein expression (Fig. 4; Table V) also corresponded with mRNA expression (Table IV), indicating 
A

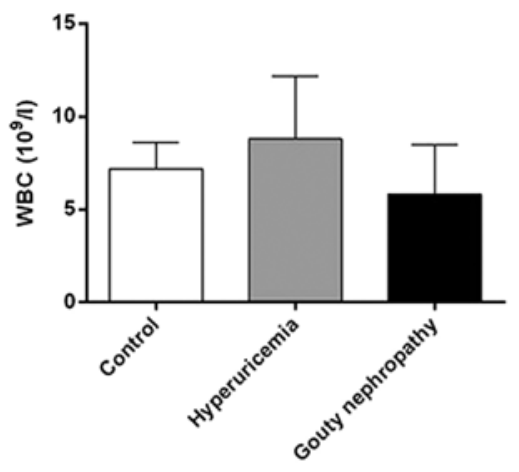

C

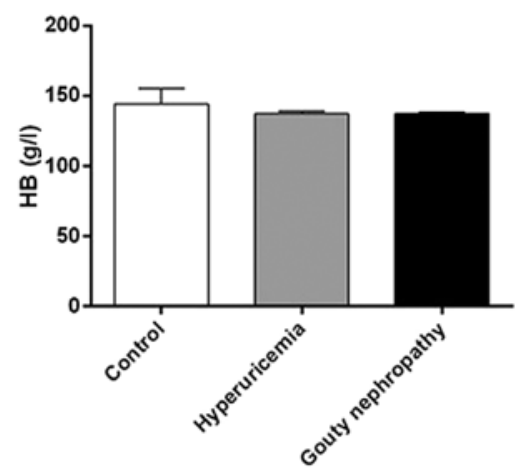

E

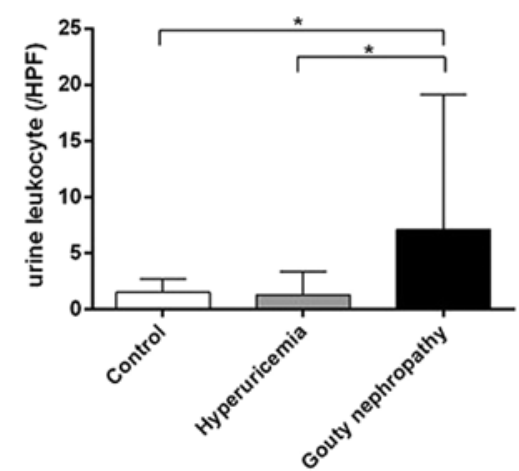

B

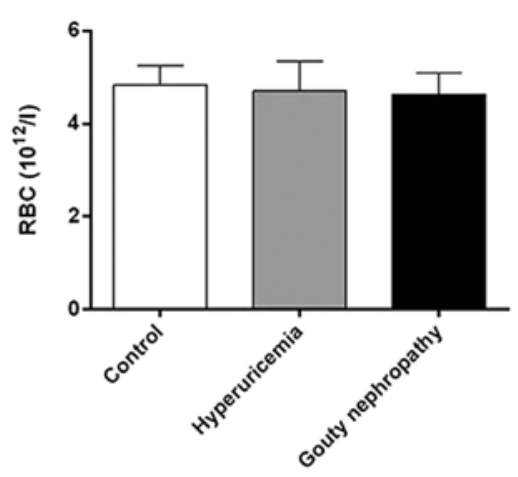

D

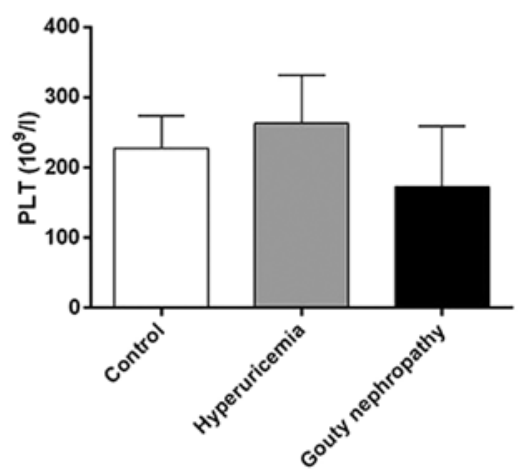

$F$

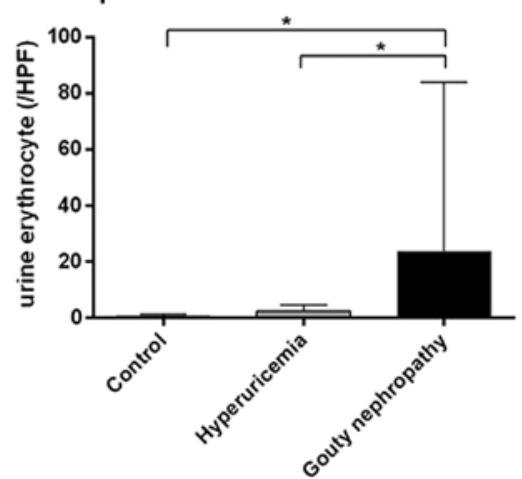

Figure 2. Organ function indicators of enrolled patients, including blood and urine analysis. (A) WBC, (B) RBC, (C) HB, (D) PLT, (E) urine leukocytes and $(\mathrm{F})$ urine erythrocytes levels of patients in the control, hyperuricemia and gouty nephropathy groups. ${ }^{*} \mathrm{P}<0.05$, with comparisons indicated by lines. WBC, white blood cell; RBC, red blood cell; HB, hemoglobin; PLT, platelets; HPF, high power field.

the important role of the $\mathrm{NLRP}_{3}$ inflammasome in gouty nephropathy.

Expression of $I L-1 \beta$ and IL-18 is increased in the hyperuricaemia and gouty nephropathy group. IL-1 $\beta$ and IL-18 levels were significantly higher in the hyperuricaemia and gouty nephropathy groups compared with the control group (Table VI). IL-1 $\beta$ expression was significantly elevated in the gouty nephropathy group compared with the hyperuricaemia group (Table VI). The increased expression of inflammatory factors demonstrated that the $\mathrm{NLRP}_{3}$ inflammasome was associated with gouty nephropathy.

\section{Discussion}

Uric acid is involved in the pathogenesis of kidney disease. Increasing evidence supports uric acid as a cause or exacerbating factor for kidney fibrosis and progressive CKD with an elevated serum uric acid level independently predicting the development of CKD $(17,18)$. Elevated uric acid levels induce oxidative stress and endothelial dysfunction, resulting in the development of both systemic and glomerular hypertension $(19,20)$. It is well established that uric acid forms urate crystals in sufficient concentrations to block renal collecting tubes, which causes interstitial nephritis, gouty nephropathy and interstitial fibrosis (21). Furthermore, the kidney damage caused by uric acid is the result of this sort of obstruction and crucially, it can be exacerbated by the inflammatory response that it initiates. Uric acid activates cytoplasmic phospholipase A2 and inflammatory transcription factor $\mathrm{NF}-\kappa \mathrm{B}$, which increases the production of various systemic cytokines, including tumour necrosis factor- $\alpha$, kidney monocyte chemotactic protein 1 and blood vessel COX-2 $(22,23)$. In addition, hyperuricaemia aggravates kidney injury by 
A
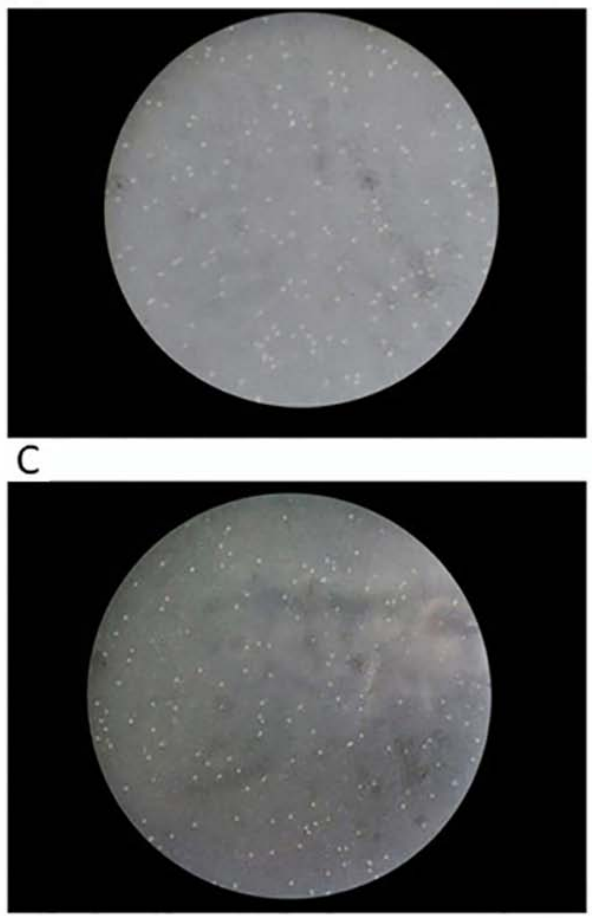

B

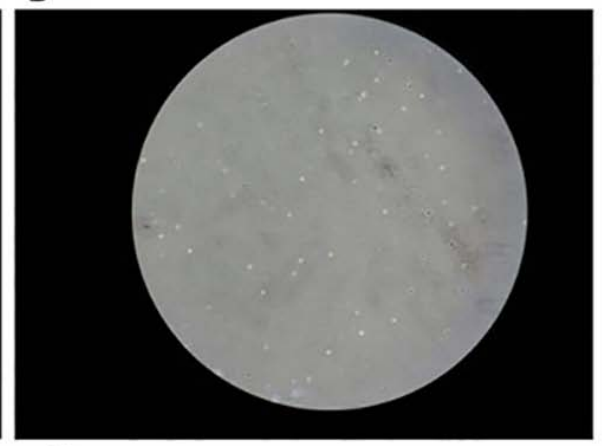

D

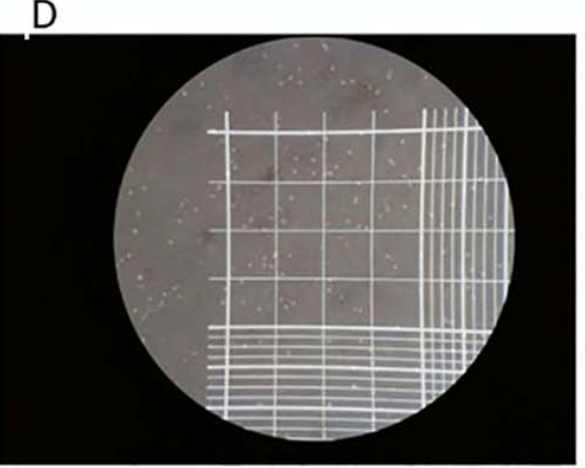

Figure 3. Representative images of trypan blue staining of peripheral blood mononuclear cells (magnification, x10). Trypan Blue staining test was used to confirmed the cell viability, followed by the counting of cells. Trypan blue staining can stain dead cells blue, whilst the live cells are not colored. (A) Densely distributed colorless transparent particles under the microscope, representing live cells. (B) Scattered distribution of colorless transparent particles, showing fewer living cells (C) Densely distributed colorless transparent particles, representing live cells. (D) Cell counting schematic. The area of every grid is 1.0 mm ${ }^{2}$ and the volume is $0.1 \mu$ l. Cells in the double-lined area were counted as red blood cells, whereas cells in the single-lined area counted as white blood cells. Magnification, $\mathrm{x} 10$.

Table III. Peripheral blood mononuclear cells count.

\begin{tabular}{lcc}
\hline Group & $\mathrm{n}$ & Count $\left(10^{5}\right)$ \\
\hline Control & 15 & $33.047 \pm 22.755$ \\
Hyperuricemia & 15 & $43.627 \pm 18.317$ \\
Gouty nephropathy & 15 & $26.567 \pm 13.506$ \\
\hline
\end{tabular}

recruiting IL-1 $\beta$-secreting macrophages, activating $\mathrm{NLRP}_{3}$ inflammasomes in macrophages and promoting chemokine secretion in proximal tubular cells (24). Uric acid, in its soluble form, is responsible for increasing the production of IL-1 $\beta$ in an $\mathrm{NLRP}_{3}$-dependent manner and is associated with kidney damage (25).

The inflammatory response is closely associated with the innate immune system. Intracellular nucleotide-binding oligomerization domain-like receptors form a group of pattern recognition receptors that are involved in a wide variety of innate host immune responses when stimulated by pathogen-associated molecular patterns dangerous-associated molecular patterns (DAMPs) (26). One of the most thoroughly investigated members is the intracellular pattern recognition receptor $\mathrm{NLRP}_{3}$ inflammasome complex consisting of $\mathrm{NLRP}_{3}$, ASC and caspase-1 (27). Upon activation, $\mathrm{NLRP}_{3}$ oligomerizes via homotypic interactions between NACHT domains and raises ASC and caspase to form an inflammatory complex, resulting in activated caspase-1. Activated caspase-1 cleaves intracellular IL-1 $\beta$ and IL-18 precursors to form mature
IL-1 $\beta$ and IL-18, which are secreted into the extracellular matrix (Fig. 5) (28). The inflammasome is understood to have a fundamental role in the progression of autoinflammatory diseases and to have additional roles in infection control, the progression of immune pathologies and the recognition of tissue damage. $\mathrm{NLRP}_{3}$ inflammasome is activated in response to a variety of infectious stimuli or by cellular stress caused by various sterile danger signals, including high concentrations of extracellular ATP, a decrease in extracellular osmolality or $\mathrm{pH}$, crystals of MSU or cholesterol and the degradation of extracellular matrix components (27).

In the present study, the concentration of uric acid and creatinine as well as the expression of mRNA and protein in $\mathrm{NLRP}_{3}$ inflammasomes including $\mathrm{NLRP}_{3}$ mRNA, ASC mRNA, caspase-1 mRNA and ASC protein levels increased in the hyperuricaemia and gouty nephropathy groups compared with the control group. Importantly, the elevated levels of IL-1 $\beta$ and IL-18 indicated that $\mathrm{NLRP}_{3}$ inflammasomes were activated in hyperuricaemia and gouty nephropathy. The expression of ASC and caspase-1 mRNA and protein was higher in the gouty nephropathy group than in the hyperuricaemia group with the level of IL-1 $\beta$ also being significantly increased. The results indicated that the $\mathrm{NLRP}_{3}$ inflammasome was activated and induced the polymerization of the adaptor molecule ASC, leading to the production of IL-1 $\beta$. Taken together, these results indirectly indicated that the $\mathrm{NLRP}_{3}$ inflammasome has a pivotal role in the progression of gouty nephropathy.

Although the specific activation mechanism of the $\mathrm{NLRP}_{3}$ inflammasome is unclear, recent studies have proposed three hypotheses regarding the activation of $\mathrm{NLRP}_{3}$ inflammasomes: 


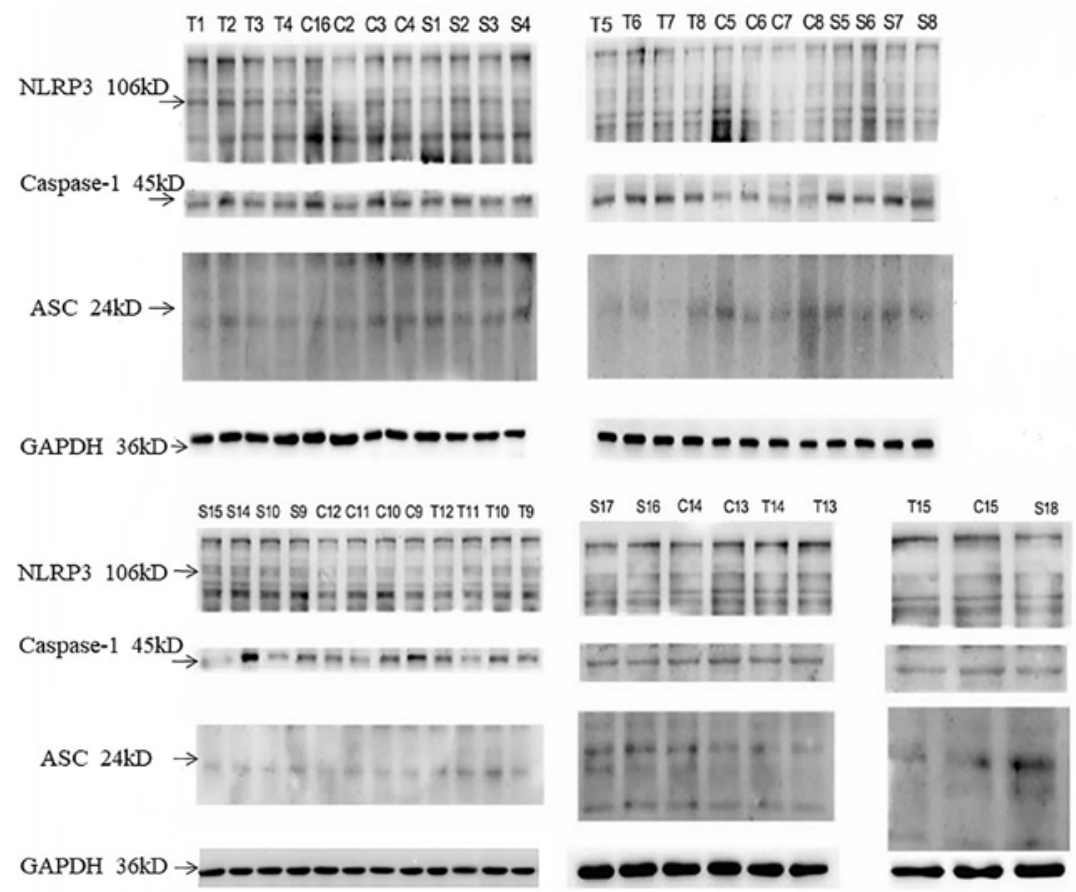

Figure 4. Expression of $\mathrm{NLRP}_{3}, \mathrm{ASC}$ and caspase-1 protein in peripheral blood mononuclear cells. Representative western blots of peripheral blood mononuclear cells isolated from control, hyperuricemia and gouty nephropathy patients. $\mathrm{NLRP}_{3}$, Nod-like receptor protein 3; ASC, apoptosis-associated speck like protein; C, control group; T, hyperuricemia group; S, gouty nephropathy group.

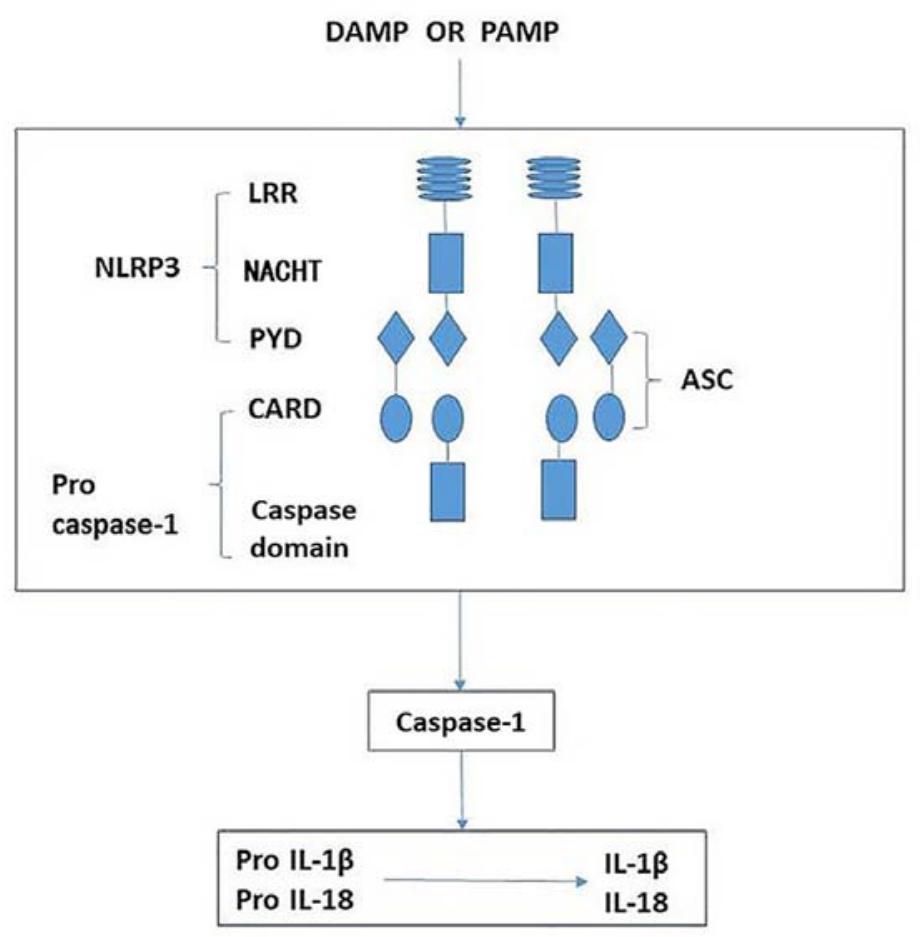

Figure 5. Schematic demonstrating the activation of $\mathrm{NLRP}_{3}$ leading to release of proinflammatory factors. The $\mathrm{NLRP}_{3}$ inflammasome is comprised of $\mathrm{NLRP}_{3}, \mathrm{ASC}$ and caspase-1. When sensing danger signals, including PAMP and DAMP, NLRP ${ }_{3}$ oligomerizes via homotypic interactions between NACHT domains. The PYD of $\mathrm{NLRP}_{3}$ is then exposed for interaction with the PYD of ASC. The CARD of ASC in turn recruits pro-caspase-1 through CARD-CARD interactions, resulting in activated caspase-1. Activated caspase- 1 then cleaves intracellular IL-1 $\beta$ and IL-18 precursors to form mature IL-1 $\beta$ and IL- 18 , which are secreted into the extracellular matrix. $\mathrm{NLRP}_{3}$, nucleotide-binding domain and leucine-rich repeat protein 3; ASC, apoptosis-associated speck like protein; LRR, leucine-rich repeat; NACHT, nucleotide-binding and oligomerization domain; PYD, pyrin domain; CARD, caspase recruitment domain; IL-1 $\beta$, interleukin-1 $\beta$; IL-18, interleukin-18; PAMP, pattern-associated molecular pattern; DAMP, danger-associated molecular pattern.

Firstly, all bacteria, viruses, particles or crystals can stimulate cells to produce reactive oxygen species, which can activate the $\mathrm{NLRP}_{3}$ inflammasome (29). In the course of oxidative stress, thioredoxin, following its own oxidation, releases 
Table IV. Expression of $\mathrm{NLRP}_{3}, \mathrm{ASC}$ and caspase-1 mRNA in peripheral blood mononuclear cells.

\begin{tabular}{lcccc}
\hline Group & $\mathrm{n}$ & $\mathrm{NLRP}_{3}$ & ASC & Caspase-1 \\
\hline Control & 15 & $23.54 \pm 1.19$ & $25.88 \pm 1.18$ & $26.99 \pm 1.43$ \\
Hyperuricemia & 15 & $26.05 \pm 1.89^{\mathrm{a}}$ & $27.49 \pm 3.56^{\mathrm{a}}$ & $28.05 \pm 2.41^{\mathrm{a}}$ \\
Gouty nephropathy & 15 & $25.43 \pm 1.66^{\mathrm{a}}$ & $32.21 \pm 3.01^{\mathrm{a}, \mathrm{c}}$ & $30.80 \pm 2.80^{\mathrm{a}, \mathrm{b}}$ \\
\hline
\end{tabular}

Values are presented as the mean \pm standard deviation. ${ }^{\mathrm{a}} \mathrm{P}<0.01$ vs. the control group; ${ }^{\mathrm{b}} \mathrm{P}<0.05$ and ${ }^{\mathrm{c}} \mathrm{P}<0.01$ vs. the hyperuricemia group; $\mathrm{NLRP}_{3}$, nucleotide-binding domain and leucine-rich repeat protein 3; ASC, apoptosis-associated speck like protein.

Table V. Expression of $\mathrm{NLRP}_{3}$, ASC, caspase-1 protein in peripheral blood mononuclear cells.

\begin{tabular}{lcccc}
\hline Group & $\mathrm{n}$ & $\mathrm{NLRP}_{3} / \mathrm{GAPDH}$ & ASC/GAPDH & Caspase-1/GAPDH \\
\hline Control & 15 & $0.45 \pm 0.18$ & $0.22 \pm 0.08$ & $0.57 \pm 0.16$ \\
Hyperuricemia & 15 & $0.55 \pm 0.12$ & $0.26 \pm 0.11 \mathrm{a}$ & $0.57 \pm 0.16$ \\
Gouty nephropathy & 15 & $0.60 \pm 0.16^{\mathrm{b}}$ & $0.47 \pm 0.19^{\mathrm{a}, \mathrm{c}}$ & $0.78 \pm 0.15^{\mathrm{b}, \mathrm{c}}$ \\
\hline
\end{tabular}

Values are presented as the mean \pm standard deviation, GAPDH was used as an internal reference. ${ }^{\mathrm{a} P}<0.05$ and ${ }^{\mathrm{b}} \mathrm{P}<0.01$ vs. the control group; ${ }^{\mathrm{c}} \mathrm{P}<0.05$ vs. the hyperuricemia group. NLRP3, nucleotide-binding domain and leucine-rich repeat protein 3; ASC, apoptosis-associated speck like protein.

Table VI. Expression of IL-1 $\beta$ and IL-18 in plasma of patients.

\begin{tabular}{llll}
\hline Group & $\mathrm{n}$ & $\mathrm{IL}-1 \beta(\mathrm{pg} / \mathrm{ml})$ & $\mathrm{IL}-18(\mathrm{pg} / \mathrm{ml})$ \\
\hline Control & 15 & $28.41 \pm 14.05$ & $323.35 \pm 96.16$ \\
Hyperuricemia & 15 & $55.19 \pm 16.79^{\mathrm{a}}$ & $718.16 \pm 211.42^{\mathrm{a}}$ \\
Gouty nephropathy & 15 & $81.20 \pm 17.63^{\mathrm{a}, \mathrm{b}}$ & $870.67 \pm 371.13^{\mathrm{a}}$ \\
\hline
\end{tabular}

Values are mean \pm standard deviation. ${ }^{\mathrm{a}} \mathrm{P}<0.01$ vs. control group; ${ }^{\mathrm{b}} \mathrm{P}<0.01$ vs. hyperuricemia group. IL-1 $\beta$, interleukin-1 $\beta$; IL-18, interleukin-18.

thioredoxin-interacting protein, activating the $\mathrm{NLRP}_{3}$ inflammasome (30). Secondly, crystals or particles, such as MSU, silicon dioxide, amyloid and cholesterol crystals, are phagocytosed by macrophages into lysosomes. This accumulation causes lysosomal rupture and the release of cathepsin B and other proteases, inducing the activation of the $\mathrm{NLRP}_{3}$ inflammasome $(31,32)$ Finally, stimulation induces cells to release ATP on the cell surface of the $\mathrm{P} 2 \mathrm{X} 7$ purine receptor, triggering the potassium ion outflow mechanism and calcium wave transport mechanism leading to mitochondrial damage. This releases mitochondrial DNA (mtDNA) and activates the $\mathrm{NLRP}_{3}$ inflammasome $(33,34)$. Previous studies have demonstrated that $\mathrm{NLRP}_{3}$ can identify uric acid crystals and other dangerous signals $(3,35)$. Uric acid crystals trigger IL-1 $\beta$-mediated inflammation by activating the $\mathrm{NLRP}_{3}$ inflammasome via the aforementioned three mechanisms (36).

The present study hypothesized that MSU acts as an endogenous DAMP, contributing to the progression of the $\mathrm{NLRP}_{3}$ inflammasome in hyperuricaemia and gouty nephropathy. When high levels of uric acid in the body cause uric acid crystals to be deposited in tissues, macrophages directly phagocytose urate crystals, which are too large to be efficiently cleared and are likely to induce the production of ROS on their way to becoming lysosomes (9). Following the phagocytosis of MSU, macrophages form phagocytic bodies and the subsequent intracellular rupture of these bodies releases cathepsin B, which activates the $\mathrm{NLRP}_{3}$ inflammasome (37). MSU also activates the $\mathrm{NLRP}_{3}$ inflammasome by causing intracellular potassium efflux (38), leading to mitochondrial damage. Furthermore, by releasing mtDNA, the maturation and release of IL-1 $\beta$ and IL-18 is stimulated. A previous study determined that uric acid activates $\mathrm{NLRP}_{3}$ inflammasomes via mitochondrial ROS production and their results demonstrated the direct role of hyperuricaemia in activating $\mathrm{NLRP}_{3}$ inflammasomes in macrophages (39). Berberine may influence the $\mathrm{NLRP}_{3}$ inflammasome and be involved in MSU crystal-induced innate immune responses, attenuating the expression of $\mathrm{NLRP}_{3}$, further inhibiting the downstream signalling of molecular IL-1 $\beta$ and eventually having a role in the treatment of gouty arthritis (40). The present study observed that gouty nephropathy is associated with elevated levels of uric acid in serum and high expression of $\mathrm{NLRP}_{3}, \mathrm{ASC}$ and caspase-1 mRNA and protein, as well as IL-1 $\beta$ and IL-18 expression. The present results indicated that uric acid-induced $\mathrm{NLRP}_{3}$ inflammasomes are associated with gouty nephropathy with the underlying mechanism potentially involving one of the aforementioned three hypotheses.

The present study has limitations as the exact association between $\mathrm{NLRP}_{3}$ inflammasomes and gouty nephropathy was not elucidated. Further studies are therefore required to explore the role of the $\mathrm{NLRP}_{3}$ inflammasome in humans and in vivo. In addition to the need for large clinical trials, more studies are required to better understand the biology of uric acid. To the best of our knowledge, clinical study into the regulation mechanism of the $\mathrm{NLRP}_{3}$ inflammasome signalling pathway with regards to gouty nephropathy has not been performed. 
In conclusion, the present study demonstrated that the $\mathrm{NLRP}_{3}$ inflammasome was associated with the progression of hyperuricaemia and gouty nephropathy, leading to the inflammatory response and renal damage. Although the precise mechanism of the $\mathrm{NLRP}_{3}$ inflammasome signalling pathway and the means by which MSU triggers the inflammasome remains a matter of debate, the importance of the $\mathrm{NLRP}_{3}$ inflammasome in gouty nephropathy has been established. The present study proposes that future therapeutic strategies for gouty nephropathy should be based on blocking uric acid or inhibiting its activation of the $\mathrm{NLRP}_{3}$ inflammasome. Further studies will be required to explore the mechanism of how uric acid activates the $\mathrm{NLRP}_{3}$ inflammasome to allow for the early diagnosis of gouty nephropathy.

\section{Acknowledgements}

Not applicable.

\section{Funding}

The present study was supported by a grant from the Shenzhen ScienceandTechnology ResearchandDevelopmentFundamental Research Project (grant no. JCYJ20160427191440905).

\section{Availability of data and materials}

The datasets used and/or analyzed during the current study are available from the corresponding author on reasonable request.

\section{Authors' contributions}

YZZ and XLS designed the experiments, analyzed the data and wrote the manuscript. YPX and YZZ performed the experiments. FJG and ASZ analyzed and interpreted the data. JHC contributed to the design of the experimental methods, wrote the manuscript and approved the version to be published and agreed to be accountable for all aspects of the work. All authors read and approved the final manuscript.

\section{Ethics approval and consent to participate}

The present study was approved by the Ethics Committee of the Affiliated Bao'an Hospital of Shenzhen (approval no. BYL2016001) and written informed consent was obtained from all participants.

\section{Patient consent for publication}

Not applicable.

\section{Competing interests}

The authors declare that they have no competing interests.

\section{References}

1. Ryu ES, Kim MJ, Shin HS, Jang YH, Choi HS, Jo I, Johnson RJ and Kang DH: Uric acid-induced phenotypic transition of renal tubular cells as a novel mechanism of chronic kidney disease. Am J Physiol Renal Physiol 304: F471-F480, 2013.
2. Siu YP, Leung KT, Tong MK and Kwan TH: Use of allopurinol in slowing the progression of renal disease through its ability to lower serum uric acid level. Am J Kidney Dis 47: 51-59, 2006.

3. Ghaemi-Oskouie F and Shi Y: The role of uric acid as an endogenous danger signal in immunity and inflammation. Curr Rheumatol Rep 13: 160-166, 2011. [J].

4. Roddy E, Zhang W and Doherty M: The changing epidemiology of gout. Nat Clin Pract Rheumatol 3: 443-449, 2007.

5. Kim IY, Lee DW, Lee SB and Kwak IS: The role of uric acid in kidney fibrosis: Experimental evidences for the causal relationship. BioMed Res Int 2014: 638732, 2014.

6. Saito I, Saruta T, Kondo K, Nakamura R, Oguro T, Yamagami K, Ozawa $\mathrm{Y}$ and Kato E: Serum uric acid and the renin-angiotensin system in hypertension. J Am Geriatr Soc 26: 241-247, 1978.

7. Gao L, Shen F and Lii YS: Effects of NLRP inflammasome on cerebral ischemia reperfusion injury. J Shanghai Jiaotong University Medical Science 35: 1896-1899, 2015.

8. Heneka MT, Kummer MP, Stutz A, Delekate A, Schwartz S, Vieira-Saecker A, Griep A, Axt D, Remus A, Tzeng TC, et al: $\mathrm{NLRP}_{3}$ is activated in Alzheimer's disease and contributes to pathology in APP/PS1 mice. Nature 493: 674-678, 2013.

9. Tschopp J and Schroder K: NLRP ${ }_{3}$ inflammasome activation: The convergence of multiple signalling pathways on ROS production? Nat Rev Immunol 10: 210-215, 2010.

10. Zheng SC, Zhu XX, Xue Y, Zhang LH, Zou HJ, Qiu JH and Liu Q: Role of the $\mathrm{NLRP}_{3}$ inflammasome in the transient release of IL-1 $\beta$ induced by monosodium urate crystals in human fibroblast-like synoviocytes. J Inflamm (Lond) 12: 30, 2015

11. Schiltz C, Lioté F, Prudhommeaux F, Meunier A, Champy R, Callebert $\mathrm{J}$ and Bardin T: Monosodium urate monohydrate crystal-induced inflammation in vivo: Quantitative histomorphometric analysis of cellular events. Arthritis Rheum 46: $1643-1650,2002$

12. Xu X, Hu J, Song N, Chen R, Zhang T and Ding X: Hyperuricemia increases the risk of acute kidney injury: A systematic review and meta-analysis. BMC Nephrol 18: 27-41, 2017.

13. Wallace SL, Robinson H, Masi AT, Decker JL, McCarty DJ and Yü TF: Preliminary criteria for the classification of the acute arthritis of primary gout. Arthritis Rheum 20: 895-900, 1977.

14. Zhang XX and Sun WF, Hou Y, Liu YW and He Y: Clinical observation on fufang tufuling keli treating 40 cases of hyperuricemia: a randomize-controlled study. J Tradit Chin Med 57: $41-45,2016$.

15. Livak KJ and Schmittgen TD: Analysis of relative gene expression data using real-time quantitative PCR and the $2(-\Delta \Delta \mathrm{C}(\mathrm{T}))$ method. Methods 25: 402-408, 2001.

16. Levey AS, de Jong PE, Coresh J, El Nahas M, Astor BC, Matsushita K, Gansevoort RT, Kasiske BL and Eckardt KU: The definition, classification, and prognosis of chronic kidney disease: A KDIGO Controversies Conference report. Kidney Int 80: 17-28, 2011.

17. Weiner DE, Tighiouart H, Elsayed EF, Griffith JL, Salem DN and Levey AS: Uric Acid and incident kidney disease in thy community. J Am soc Nephriol 19: 1204-1211, 2008.

18. Weiner DE, Tighiouart H, Elsayed EF, Griffith JL, Salem DN and Levey AS: Uric acid and incident kidney disease in the community. J Am Soc Nephrol 19: 1204-1211, 2008.

19. Sánchez-Lozada LG, Soto V, Tapia E, Avila-Casado C, Sautin YY, Nakagawa T, Franco M, Rodríguez-Iturbe B and Johnson RJ: Role of oxidative stress in the renal abnormalities induced by experimental hyperuricemia. Am J Physiol Renal Physiol 295: F1134-F1141, 2008.

20. Kang DH, Nakagawa T, Feng L, Watanabe S, Han L, Mazzali M, Truong L, Harris R and Johnson RJ: A role for uric acid in the progression of renal disease. J Am Soc Nephrol 13: 2888-2897, 2002.

21. Liu LQ: Pathogenesis of hyperuric acid nephropathy. J Clin Nephrol 11: 53-55, 2011

22. Kanellis J, Watanabe S, Li JH, Kang DH, Li P, Nakagawa T, Wamsley A, Sheikh-Hamad D, Lan HY, Feng L, et al: Uric acid stimulates monocyte chemoattractant protein-1 production in vascular smooth muscle cells via mitogen-activated protein kinase and cyclooxygenase-2. Hypertension 41: 1287-1293, 2003. $[\mathrm{J}]$.

23. Prasad Sah OS and Qing YX: Associations between hyperuricemia and chronic kidney disease: A Review. Nephrourol Mon 7: e27233, 2015.

24. Gul A and Zager P: Does altered uric acid metabolism contribute to diabetic kidney disease pathophysiology? Curr Diab Rep 18: 18,2018 . 
25. Braga TT, Forni MF, Correa-Costa M, Ramos RN, Barbuto JA Branco P, Castoldi A, Hiyane MI, Davanso MR, Latz E, et al: Soluble uric acid activates the $\mathrm{NLRP}_{3}$ inflammasome. Sci Rep 7: 39884, 2017.

26. Isaka Y, Takabatake Y, Takahashi A, Saitoh T and Yoshimori T: Hyperuricemia-induced inflammasome and kidney diseases. Nephrol Dial Transplant 31: 890-896, 2016.

27. Baroja-Mazo A, Martín-Sánchez F, Gomez AI, Martínez CM, Amores-Iniesta J, Compan V, Barberà-Cremades M, Yagüe J, Ruiz-Ortiz E, Antón J, et al: The $\mathrm{NLRP}_{3}$ inflammasome is released as a particulate danger signal that amplifies the inflammatory response. Nat Immunol 15: 738-748, 2014.

28. Xia M, Boini KM, Abais JM, Xu M, Zhang Y and Li PL: Endothelial $\mathrm{NLRP}_{3}$ inflammasome activation and enhanced neointima formation in mice by adipokine visfatin. Am J Pathol 184: 1617-1628, 2014.

29. Zhou R, Tardivel A, Thorens B, Choi I and Tschopp J: Thioredoxin-interacting protein links oxidative stress to inflammasome activation. Nat Immunol 11: 136-140, 2010.

30. Martinon F: Signaling by ROS drives inflammasome activation. Eur J Immunol 40: 616-619, 2010.

31. Gasse P, Riteau N, Charron S, Girre S, Fick L, Pétrilli V, Tschopp J, Lagente V, Quesniaux VF, Ryffel B, et al: Uric acid is a danger signal activating NALP3 inflammasome in lung injury inflammation and fibrosis. Am J Respir Crit Care Med 179 903-913, 2009.

32. Jiang H, Yan YQ, Jiang $W$ and Zhou RB: $N_{R R P}$ inflammasome: Activation, regulation, and role in diseases. Sci Sin Vitae 47: 125-131, 2017.
33. Zhou R, Yazdi AS, Menu P and Tschopp J: A role for mitochondria in $\mathrm{NLRP}_{3}$ inflammasome activation. Nature 469: 221-225, 2011.

34. Jin-Feng LI, Xie D, Ping-Ping HE, Tang YY, Yu-Lin TU and Yin K: Research advances of the $\mathrm{NLRP}_{3}$ inflammasome and metabolic disease. Prog Biochem Biophys 41: 425-434, 2014.

35. Martinon F, Pétrilli V, Mayor A, Tardivel A and Tschopp J: Gout-associated uric acid crystals activate the NALP3 inflammasome. Nature 440: 237-241, 2006.

36. Xu LL: $\mathrm{NLRP}_{3}$ inflammasomes and kidney diseases. J Nephrol Dialy Transpl 20: 554-558, 2011.

37. Hornung V, Bauernfeind F, Halle A, Samstad EO, Kono H, Rock KL, Fitzgerald KA and Latz E: Silica crystals and aluminum salts activate the NALP3 inflammasome through phagosomal destabilization. Nat Immunol 9: 847-856, 2008.

38. Stutz A, Golenbock DT and Latz E: Inflammasomes: Too big to miss. J Clin Invest 119: 3502-3511, 2009.

39. Kim SM, Lee SH, Kim YG, Kim S-Y, Seo JW, Choi YW, Kim DJ, Jeong KH, Lee TW, Ihm CG, et al: Hyperuricemia-induced $\mathrm{NLRP}_{3}$ activation of macrophage contributes to the progression of diabetic nephropathy. Am J Physiol-renal 308: 993-1003, 2015.

40. Liu YF, Wen CY, Chen Z, Wang Y, Huang Y and Tu SH: Effects of berberine on $\mathrm{NLRP}_{3}$ and IL- $1 \beta$ expressions in monocytic THP-1 cells with monosodium urate crystals-induced inflammation. Biomed Res Int 2016: 2503703, 2016.

(c) (1) () This work is licensed under a Creative Commons

Attribution-NonCommercial-NoDerivatives 4.0 International (CC BY-NC-ND 4.0) License. 